\title{
Therapeutic Innovations in Cancer, Review
}

\author{
Hajra Haneef ${ }^{1}$, Nazar-ul-Islam ${ }^{2}$ and Khalid Mahmood ${ }^{2}$ \\ ${ }^{1}$ Ex-student of Quaid-e-Azam University, Islamabad,Pakistan \\ ${ }^{2}$ Biological Production Division, National Institute of Health, Chak shahzad, Pakistan
}

Received: 非 August 30, 2018; Published: 䟧 September 10, 2018

*Corresponding author: Hajra Haneef, Ex-student of Quaid-e-Azam University, Islamabad, Pakistan

\begin{abstract}
Therapies in field of tumor are still new. A lot of avenues have to discover, various are still in non-clinical trials. Some achieve success in their targets in clinical fields. But now, the time turn, for production of vaccine against cancer and their trials on human. No doubt, this production is time consuming and expensive treatment in these days, but as it progress, it will be in human victory as like other medications. Hence, Cancer vaccines represent a promising avenue for cancer immunotherapy.
\end{abstract}

Keywords: Cancer; Monoclonal Antibodies; Adoptive Therapy; Vaccines

\section{Introduction}

Cancer is the second leading cause of death in the world after cardiovascular diseases. Cancer develops when normal cells in a particular part of the body begin to grow out of control. Almost all studies showed a correlation of fatigue with depression and with anxiety in cancer. There are different types of cancers. Different types of cancer cells can behave differently. Generally, cancer cells develop from normal cells due to damage of DNA. During the 1970s, Scientists identified oncogenes and tumor suppressor genes that are damaged by chemicals (carcinogens), radiation, viruses and also inherited from ancestors, responsible for cancer. Most carcinogens damage the DNA, which led to abnormal growth of cells. Cancer cells with damaged DNA do not die, where as normal cells with damaged DNA die [1,2]. In 2018, 1,735,350 new cancer cases and 609,640 cancer deaths are projected to occur in the United States [3]. One study in 2016, showed that in Lahore, Pakistan the AgeStandardized Incidence Rates (ASIR) was higher in women than in men. Among women and men, breast cancer and prostate cancer, respectively, were the leading cancer types $[4,5]$.

\section{Cancer Immunotherapy}

Cancer immunotherapy including monoclonal antibodies (mAbs), the immune checkpoint therapy, adoptive cancer therapy and cancer vaccines has revolutionized traditional cancer treatment [6].

\section{Monoclonal Antibodies}

Immunotherapy aims to boost the immune system in its fight against cancer. The ways to accomplish this, are many and still in the process of research; one of them uses monoclonal antibodies to interfere with cancer cells' ability to fool the immune system into overlooking them. Especially useful against melanoma and smokinginduced lung cancer, this method is often referred to as 'checkpoint blockade' [7]. The use of monoclonal antibodies for the therapy of cancer is one of the major contributions of tumor immunology to cancer patients. Currently, there are several hundred mAb-based products at different stages of preclinical studies and clinical trials for use in oncology $[8,9]$. Many mAbs have been approved by the FDA to treat different types of solid tumors and most of them were developed to recognize almost the same tumor targets like HER2, EGFR, VEGF/R, CTL4 and PD1-PD-L1 and less extensively GD2 and RANKL with the purpose to block oncogenic pathways and the formation of new blood vessels, to modulate the immune response against tumor cells and to regulate osteoclast function and deliver cytotoxic drugs to the tumor cells. Clinical trials showed that the use of mAbs may improve the overall survival in many cancerous conditions as single agent or in combination with standard chemotherapy and, with the exception of Bevacizumab that was withdrawn in 2011 for the treatment of metastatic breast cancer, the rest of them are still available in clinical practice [10].

\section{Immune Checkpoint Therapy}

In cancer, immune checkpoint pathways are often activated to inhibit the nascent anti-tumor immune response. Immune checkpoint therapies act by blocking or stimulating these pathways and enhance the body's immunological activity against tumors. Cytotoxic T lymphocyte-associated molecule-4 (CTLA-4), programmed cell death receptor-1 (PD-1), and programmed cell 
death ligand-1(PD-L1) are the most widely studied and recognized inhibitory checkpoint pathways [11].

\section{Adoptive Cancer Therapy}

(Adoptive cell transfer (ACT), Adoptive T cell immunotherapy) Adoptive T cell immunotherapy studies have provided strong proof of principle that antigen-specific CD8+ T cell responses to cancer can mediate dramatic cancer regressions. However, adoptive therapy is cumbersome and expensive, and difficult in the current regulatory environment [12]. Another promising strategy is using alternating cells, such as B cells and synthetic antigen presenting cells (APCs) that mimic the functions of natural APCs and are much easier to manufacture. Immunotherapy based on the adoptive transfer of naturally occurring or gene-engineered T cells can mediate tumour regression in patients with metastatic cancer $[13,14]$.

Classical treatments for cancers remind us of surgery, chemotherapy, and radiation therapy. In the last two decades, targeted therapies like imatinib (Gleevec) and trastuzumab (Herceptin)-drugs that target cancer cells-have established themselves as standard treatments for some cancers. Now, the world is likely to witness the miracle of immunotherapy. Vaccination strategies are extremely cost effective, alleviating the socioeconomic problems of prohibitively high drug costs $[15,16]$.

\section{The Current Manuscript is Focused Primarily on Vaccine Therapy}

The goal of a therapeutic cancer vaccine is to induce the activation and proliferation of $\mathrm{T}$ cells and can induce interferon $\gamma($ IFN- $\gamma$ ) secretion, which elicits the expression of programmed death ligand 1 (PD-L1) in the tumor and thus induces adaptive immune resistance $[17,18]$. Vaccines may prevent cancer by preventing the causative viral infection. Few cancer vaccines have been approved by the US Food and Drug Administration (FDA), for instance, Provenge, and T-VEC got approval for the treatment of melanoma [19,20]. A vaccine specific for tumor antigens may have wide application and utility in the prevention of recurrence in numerous different malignancies. Peptide vaccines are easily produced, standardized, and administered [21,22]. The strategy of genetically modified tumor cells with MHC class II transcriptional activator CIITA has been applied for the production of a novel generation of anti-tumor vaccine against human hepatocarcinoma that is now in clinical trial.(3). Another area for improvement in immunotherapy is dendritic vaccines.

First-in-human clinical trials of personalized cancer vaccines have shown the feasibility, safety, and immunotherapeutic activity of targeting individual tumor mutation signatures [23,24]. Neoantigen cancer vaccines (NCVs) may represent an emerging new clinical approach to treat cancer. In July 2017, two research teams simultaneously reported in Nature that melanoma patients at high risk of recurrence responded impressively to personalized "neoantigen" vaccines [25,26]. Cancer treatment is a highly dynamic field and significant advances are being made in the development of cancer treatment strategies. In contrast to conventional cancer therapeutics, techniques such as ligand or receptor based targeting, triggered release, intracellular drug targeting, gene delivery, cancer stem cell therapy, magnetic drug targeting and ultrasound mediated drug delivery, have added new modalities for cancer treatment. These techniques have led to selective detection of malignant cells leading to their eradication with minimal side effects. Advances in genome sequencing are identifying predictive biomarkers and facilitating the design of personalized vaccines that target patientspecific tumor neoantigens.

Lowering, multi-drug resistance and involving influx transportation in targeted drug delivery to cancer cells can also contribute significantly in the therapeutic interventions in cancer $[27,28]$. Cancer vaccines are attracting new interest as combination partners with other immunotherapies, in particular $\mathrm{T}$ cell checkpoint blockade approaches. Importantly, recent studies have shown that immunotherapy may work best if delivered with chemotherapy specifically, if the chemotherapy drugs are delivered first, and they are followed up with immunotherapy $[29,30]$. The Cancer Vaccine Collaborative, a joint academic clinical trials infrastructure established by LICR and the Cancer Research Institute (CRI), is about to embark on a series of trials exploring NY-ESO-1 vaccines along with ipilimumab to further investigate this important area [26].

\section{Viruses in Tumor World}

On the one hand, Virus-associated cancers, which account for more than $20 \%$ of cancers worldwide, express viral neoantigens that are strongly immunogenic [27]. While on other, A surprising weapon in the fight against cancer could be therapeutic viruses, as revealed by a team from the United Kingdom. In their experiments, they managed to use a reovirus to attack brain cancer cells while leaving healthy cells alone. Oncolytic viruses (OVs) also have shown positive outcomes for cancer treatment $[23,28]$.

\section{Bacteria in Field of Cancer}

Bacterial cancer therapy dates back to 1893. Scientists use modified Salmonella to deliver various therapeutic agents to the body, such as genes and anti-cancer medicines. Another most prominent example would be the use of Mycobacterium bovis $B C G$, the vaccine strain, in the treatment of bladder cancer $[29,30]$. Bacterial toxins too have emerged as promising cancer treatment strategy. Bacteria also used as gene delivery vehicles in solid cancer. The most potential and promising strategy is bacteria-based genedirected enzyme prodrug therapy [31,32].

\section{Nanoparticle (Liposome, Quantum Dot, and Dendrimer)-Mediated Cancer Therapy}

Scientists from China and the U.K. managed to come up with a type of "self-regulating" nanoparticle that was able to expose tumors to heat while avoiding contact with healthy tissue [23]. Nanomedicine, nanowires and nanocantilever, nanodevices can provide essential breakthroughs in the fight against cancer $[33,34]$.

\section{Conclusion}

With the current knowledge, the rapid advance of science and the arrival of biosimilars it is highly probable that this century give 
us the light to let behind the old fashion approach of treating cancer patients with unspecific chemotherapy to finally move forward to a more tailored approach based on predictive biomarkers not just for mAbs or adoptive cellular therapy but also for small molecules.

\section{Future Prospects}

a) To reach further clinical success, efforts should focus on target identification, optimization of vaccination strategies and adjuvant development.

b) Combining vaccines and immune checkpoint inhibitors to prime, expand, and facilitate effective tumor immunotherapy.

c) A detailed understanding of the mechanism of action of anti-cancer vaccination is need for the design of potent vaccine approaches that induce robust $\mathrm{T}$ cell responses

d) There is still room for improvement like expanding the biomarkers scope.

\section{References}

1. Linda F, Brown MS, Kurt Kroenke MD (2009) Cancer-Related Fatigue and Its Associations With Depression and Anxiety: A Systematic Review. Psychosomatics 50(5): 440-447.

2. Sudhakar A (2010) History of Cancer, Ancient and Modern Treatment Methods. J cancer Sci Ther 1(2): 1-4.

3. Siegel RL, Miller KD, Jemal A (2018) Cancer statistics. CA Cancer J Clin 68(1): 7-30.

4. Badar F, Mahmood S, Yusuf MA, Sultan F (2016) Epidemiology of cancers in Lahore, Pakistan, 2010-2012: a cross-sectional study. BMJ Open 6: 6.

5. Zhang H, Chen J (2018) Current status and future directions of cancer immunotherapy. Journal of Cancer 9(10): 1773-1781.

6. Islam M (2016) Waging War Against Cancer: A Peek into Immunotherapy. Journal of Pioneering Medical Sciences Blog.

7. Modjtahedi H, Ali S, Essapen S (2012) Therapeutic application of monoclonal antibodies in cancer: advances and challenges. British Medical Bulletin 104(1): 41-59.

8. Scott AM, Allison JP, Wolchok JD (2012) Monoclonal antibodies in cancer therapy. Cancer Immun 12: 14

9. Chiavenna SM, Jaworski JP, Vendrell A (2017) State of the art in anticancer mAbs. Journal of Biomedical Science 24(1): 15.

10. Marin Acevedo JA, Dholaria B, Soyano AE, Knutson KL, Chumsri S, et al. (2018) Next generation of immune checkpoint therapy in cancer: new developments and challenges. J Hematol Oncol 11(1): 39.

11. Slingluff]r CL, Speiser DE (2005) Progress and controversies in developing cancer vaccines. Journal of Translational Medicine 3(1): 18.

12. Restifo NP, Dudley ME, Rosenberg SA (2012) Adoptive immunotherapy for cancer: harnessing the $\mathrm{T}$ cell response. Nature Reviews Immunology 12(4): 269-281.

13. Guo Y, Lei K, Tang L (2018) Neoantigen Vaccine Delivery for Personalized Anticancer Immunotherapy. Front Immunol 9: 1499.
14. Huijbers EJM, Griffioen AW (2017) The revival of cancer vaccines - The eminent need to activate humoral immunity. Hum Vaccin Immunother 13(5): 1112-1114.

15. Bashir MM (2018) Car T cells - Get Own Cells to Treat Cancers. Pakistan Journal of pathology 28(4): 153-154.

16. Wang T, Wang D, Yu H, Feng B, Zhou F, et al. (2018) A cancer vaccinemediated postoperative immunotherapy for recurrent and metastatic tumors. Nature Communications 9: 1532.

17. Khong H, Willem W, Overwijk (2016) Adjuvants for peptide-based cancer vaccines. Journal for ImmunoTherapy of Cancer 4: 56.

18. Ye ZL, Qian Q, Jin HJ, Qian QJ (2018) Cancer vaccine: learning lessons from immune checkpoint inhibitors 9(2): 263-268.

19. Obara W, Kanehira M, Katagiri T, Kato R, Kato Y, et al. (2018) Present status and future perspective of peptide-based vaccine therapy for urological cancer. Cancer Sci 109(3): 550-559.

20. Roberto A (2018) B-104 Cancer vaccine: Tumor immunology meets... Immunology. Journal of Acquired Immune Deficiency Syndromes 77: 36.

21. Sahin U, Türeci Ö (2018) Personalized vaccines for cancer immunotherapy. Science 359(6382): 1355-1360.

22. Cohut M (2018) The state of cancer: Are we close to a cure? Medical News Today.

23. Aurisicchio L, Pallocca M, Ciliberto G, Palombo F (2018) The perfect personalized cancer therapy: cancer vaccines against neoantigens. Journal of Experimental \& Clinical Cancer Research 37: 86.

24. Topalian SL, Taube JM, Anders RA, Pardoll DM (2016) Mechanism-driven biomarkers to guide immune checkpoint blockade in cancer therapy. Nature Reviews Cancer 16(5): 275-287.

25. Song S, Vuai MS, Zhong M ( 2018) The role of bacteria in cancer therapy - enemies in the past, but allies at present. Infectious Agent Cancer 13: 9.

26. Dvorsky G (2017) An Innovative New Cancer Therapy Hijacks Bacteria to Fight Tumors. Gizmodo.

27. Chakrabarty AM (2003) Microorganisms and Cancer: Quest for a Therapy. J Bacteriol 185(9): 2683-2686.

28. Ryan RM, Green J, Lewis CE (2006) Use of bacteria in anti-cancer therapies. Bio Essays 28 (1): 84-94.

29. Patyar S, Joshi R, Byrav DSP, Prakash A, Medhi B, et al. (2010) Bacteria in cancer therapy: a novel experimental strategy. Journal of Biomedical Science 17(1): 21.

30. Liu Y, Miyoshi H, Nakamura M (2007) Nanomedicine for drug delivery and imaging: A promising avenue for cancer therapy and diagnosis using targeted functional nanoparticles. International Journal of Cancer 120(12): 2527-2537.

31. Ferrari M (2005) Cancer nanotechnology: opportunities and challenge. Nature Reviews Cancer 5(3): 161-171.

32. Schmidt C (2017) Personalized Cancer Vaccines Advance in the Clinic. Journal of the National Cancer Institute 109(11).

33. Junejo AR (2016) Diagnosis Techniques for Cancer Therapeutics in Pakistan By Nanotechnology: A Reciew Research Gate Online.

34. Kelly PN (2018) The Cancer Immunotherapy Revolution. Science 359(6382): 1344-1345. 


\section{ISSN: 2574-1241}

DOI: 10.26717/BJSTR.2018.08.001721

Hajra Haneef. Biomed J Sci \& Tech Res

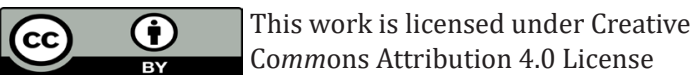

Submission Link: https://biomedres.us/submit-manuscript.php

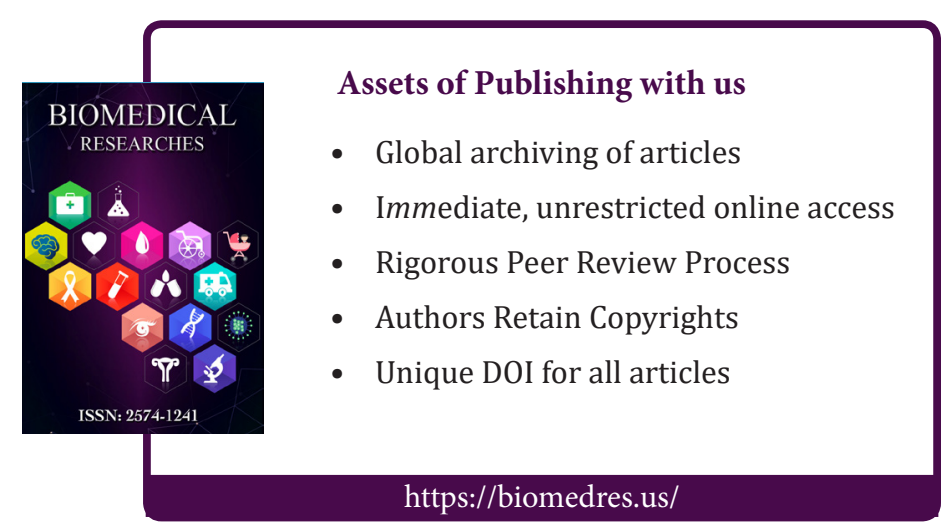

